\title{
An Artificial Neural Network-Based Pediatric Mortality Risk Score: Development and Performance Evaluation Using Data From a Large North American Registry
}

Niema Ghanad Poor ${ }^{1,2}$, MSc; Nicholas C West ${ }^{3}$, MSc; Rama Syamala Sreepada ${ }^{1,3}$, PhD; Srinivas Murthy ${ }^{1,4}$, MD; Matthias Görges ${ }^{1,3}, \mathrm{PhD}$

\footnotetext{
${ }^{1}$ Research Institute, BC Children's Hospital, Vancouver, BC, Canada

${ }^{2}$ Department of Electrical Engineering and Computer Science, Technische Hochschule Lübeck, Lübeck, Germany

${ }^{3}$ Department of Anesthesiology, Pharmacology \& Therapeutics, The University of British Columbia, Vancouver, BC, Canada

${ }^{4}$ Department of Pediatrics, The University of British Columbia, Vancouver, BC, Canada
}

\section{Corresponding Author:}

Matthias Görges, $\mathrm{PhD}$

Department of Anesthesiology, Pharmacology \& Therapeutics

The University of British Columbia

Rm V3-324, 950 West 28th Avenue

Vancouver, BC, V5Z 4H4

Canada

Phone: 16048752000 ext 5616

Fax: 16048752668

Email: mgorges@bcchr.ubc.ca

\section{Abstract}

Background: In the pediatric intensive care unit (PICU), quantifying illness severity can be guided by risk models to enable timely identification and appropriate intervention. Logistic regression models, including the pediatric index of mortality 2 (PIM-2) and pediatric risk of mortality III (PRISM-III), produce a mortality risk score using data that are routinely available at PICU admission. Artificial neural networks (ANNs) outperform regression models in some medical fields.

Objective: In light of this potential, we aim to examine ANN performance, compared to that of logistic regression, for mortality risk estimation in the PICU.

Methods: The analyzed data set included patients from North American PICUs whose discharge diagnostic codes indicated evidence of infection and included the data used for the PIM-2 and PRISM-III calculations and their corresponding scores. We stratified the data set into training and test sets, with approximately equal mortality rates, in an effort to replicate real-world data. Data preprocessing included imputing missing data through simple substitution and normalizing data into binary variables using PRISM-III thresholds. A 2-layer ANN model was built to predict pediatric mortality, along with a simple logistic regression model for comparison. Both models used the same features required by PIM-2 and PRISM-III. Alternative ANN models using single-layer or unnormalized data were also evaluated. Model performance was compared using the area under the receiver operating characteristic curve (AUROC) and the area under the precision recall curve (AUPRC) and their empirical 95\% CIs.

Results: Data from 102,945 patients (including 4068 deaths) were included in the analysis. The highest performing ANN (AUROC 0.871, 95\% CI 0.862-0.880; AUPRC 0.372, 95\% CI 0.345-0.396) that used normalized data performed better than PIM-2 (AUROC 0.805, 95\% CI 0.801-0.816; AUPRC 0.234, 95\% CI 0.213-0.255) and PRISM-III (AUROC 0.844, 95\% CI 0.841-0.855; AUPRC 0.348, 95\% CI 0.322-0.367). The performance of this ANN was also significantly better than that of the logistic regression model (AUROC 0.862, 95\% CI 0.852-0.872; AUPRC 0.329, 95\% CI 0.304-0.351). The performance of the ANN that used unnormalized data (AUROC 0.865, 95\% CI 0.856-0.874) was slightly inferior to our highest performing ANN; the single-layer ANN architecture performed poorly and was not investigated further.

Conclusions: A simple ANN model performed slightly better than the benchmark PIM-2 and PRISM-III scores and a traditional logistic regression model trained on the same data set. The small performance gains achieved by this two-layer ANN model may not offer clinically significant improvement; however, further research with other or more sophisticated model designs and better imputation of missing data may be warranted. 
(JMIR Med Inform 2021;9(8):e24079) doi: 10.2196/24079

\section{KEYWORDS}

artificial intelligence; risk assessment; decision support techniques; intensive care unit; pediatric; decision making; computer-assisted

\section{Introduction}

\section{Background}

The use of risk models in medicine enables timely and more targeted interventions for a given patient and facilitates benchmarking quality of care and conduct of clinical studies [1]. It is often necessary to quantify the severity of illness in the pediatric intensive care unit (PICU). Estimating the probability of mortality or expected length of stay from early admission data with such risk models is mainly used for quality improvement and benchmarking; however, it might enable a clinician to make objective medical decisions regarding the state of the patient, the necessary level of care, possible treatments, discharge plans, or expected costs [2-4].

PICUs are data-rich environments with a wide range of physiological variables that are responsive to interventions over short periods and outcomes that are well-defined and generally quantifiable [5]. Thus, the PICU provides fertile ground to develop and test prediction models of risks and outcomes. A score, which is quick and pragmatic to use, can enable the timely identification of adverse conditions and may be used to tailor appropriate interventions [6]. Two commonly encountered pediatric risk scores are the pediatric index of mortality 2 (PIM-2) [2] and pediatric risk of mortality III (PRISM-III) [1]. Both are derived from logistic regression models, which estimate mortality risk and have been validated with respective areas under the receiver operating characteristic curves (AUROCs) of 0.90 and 0.89 [1,7].

Increased computing capabilities, big data, and machine learning algorithms enable the application of artificial intelligence (AI) for clinical decision support [8]. Artificial neural networks (ANNs), a subtype of AI, can be used in different medical areas and have been shown to outperform physicians in diagnosis based on medical imaging or data from electronic medical records [9-12]. A recurrent neural network is a type of ANN that is most commonly used for sequential data. An ANN-based cardiac risk score, which used the recurrent neural network approach, was able to detect small changes in an electrocardiogram segment, which cannot be found by visual inspection [11]; another was used to classify clinical time series data for pediatric patients in critical care [12].

The clinical adoption of ANN-based risk models relies on gaining physicians' trust in the use of AI $[13,14]$, which may include, but is not limited to, demonstrating better performance than traditional regression approaches.

\section{Objectives}

The primary aim of this study is to examine the performance of an ANN-based approach compared to that of traditional approaches based on logistic regression models when applied to estimating the risk of mortality in children admitted to PICU with suspected sepsis. We developed an ANN model using features required in the PIM-2 and PRISM-III models to predict mortality outcomes (died or survived) in a large North American registry data set and evaluated the ANN's performance using the AUROC. We compared its performance with the benchmark PIM-2 and PRISM-III scores, as well as a logistic regression model, trained on the study data set, which used the same features as PIM-2 and PRISM-III.

\section{Methods}

\section{Study Design and Approval}

In this study, we used data from a North American PICU registry to compare the performance of an ANN model with PIM-2 and PRISM-III scores. The data set was obtained from Virtual Pediatric Systems (VPS), LLC, a registry of prospectively collected records from 130 PICUs in the United States and Canada. This is a secondary analysis of data obtained for a different purpose - to develop a simple risk stratification score for children with sepsis [6]. Ethical approval for the study was obtained from the University of British Columbia/Children's and Women's Health Centre of British Columbia Research Ethics Board (H15-01398). The requirement for written informed consent was waived by the research ethics board, as this study was a secondary analysis of registry data. This manuscript has been prepared in accordance with the guidelines for Transparent Reporting if a multivariable prediction risk model for Individual Prognosis or Diagnosis.

As sepsis diagnosis might not necessarily be known or documented at the time of admission to the PICU, we identified all children in the VPS data set whose diagnostic codes at discharge exhibited evidence of an infection, and combined with their admission to the PICU, this provides a reasonably strong indication for sepsis. This allowed us to create a representative data set of children with a high likelihood of sepsis.

\section{Study Data Set}

\section{Data Available for Analysis}

The analyzed data set included data on PICU admissions between January 1, 2009, and December 31, 2014. Data were available from 102,945 children, of whom 4068 died (mortality rate $3.95 \%$ ). Each entry included a variety of vital signs, laboratory tests, and other clinical information, including the variables required to calculate the PIM-2 and PRISM-III scores. The clinical data used in this analysis were solely from early admission to the PICU. Hence, the longer the length of stay, the less associated these predictors were with the outcome under investigation: mortality or survival at PICU discharge.

Although the variables for PIM-2 and PRISM-III were collected from the same source, these models captured data from different sampling windows. For any given PICU admission, the VPS data set provides a single measurement for each variable used 
by these 2 risk scores as required for their respective calculations.

\section{PRISM-III Variables and Sampling Window}

PRISM-III uses the highest or lowest values of systolic blood pressure, heart rate, temperature, mental status, pupillary reflexes, acidosis, $\mathrm{pH}, \mathrm{P}_{\mathrm{CO} 2}$, total carbon dioxide $\left(\mathrm{CO}_{2}\right), \mathrm{Pa}_{\mathrm{O} 2}$, glucose, potassium, creatinine, blood urea nitrogen, white blood cell count, platelet count, and prothrombin time or partial thromboplastin time [1]. Values included were measured in the first 12 hours of PICU care; laboratory variables were also considered up to 2 hours before PICU admission.

\section{PIM-2 Variables and Sampling Window}

PIM-2 uses the first recorded values of systolic blood pressure, pupillary reaction to light, $\mathrm{Pa}_{\mathrm{O} 2}$, base excess, early mechanical ventilation (yes or no), elective PICU admission (yes or no), admission following surgery (yes or no), admission following cardiopulmonary bypass, high-risk diagnoses (nine options: cardiac arrest preceding intensive care unit (ICU) admission, severe combined immune deficiency, leukemia or lymphoma after first induction, spontaneous cerebral hemorrhage, cardiomyopathy or myocarditis, hypoplastic left heart syndrome, HIV infection, liver failure as the main reason for ICU admission, or neurodegenerative disorder), and low-risk diagnoses (five options: main reason for ICU admission of asthma, bronchiolitis, croup, obstructive sleep apnea, or diabetic keto-acidosis) [2]. Values included were measured in the first hour of PICU care starting at the time of the first face-to-face meeting of the patient with a PICU team member.

Not all vital signs were collected routinely for every patient, so the data set was only sparsely populated, and the vital signs used for calculating PIM-2 and PRISM-III scores were incomplete in some cases, for example, the Glasgow Coma Score (mental status) was missing from $60.2 \%(61,976 / 102,945)$ of cases. In the calculation of both PIM-2 and PRISM-III scores, missing vital signs are taken as a sign of being normal, that is, healthy, as such tests were not ordered or performed by the PICU team [1,2]. For example, a missing Glasgow Coma Score is interpreted as indicating a normal mental status and is input to the model as such. This assumption is discussed further in the Limitations section.

\section{Preprocessing}

Preprocessing was performed in Python (v3.8.5; Python Software Foundation) to perform three tasks: (1) generate the training and test sets, (2) address missing values in the data set, and (3) generate new variables through data transformation.

\section{Generation of Training and Test Sets}

The total data set was initially divided into training and test sets using a stratified approach to ensure that the class ratio for mortality remained approximately equal for the training, test, and full data sets. ANN and logistic regression models were built on the training sets and evaluated on the test sets, and the results were compared against the PIM-2 and PRISM-III models. The overall data set was bootstrapped 100 times to generate the training and test sets.

\section{Addressing Data Missingness Through Simple Substitution}

The data set was examined for missing entries, and the missing values were imputed based on the feature type; specifically, the missing values in categorical features, such as pupillary reaction and coma status, were imputed using the most common value (mode). The missing values in numerical features, such as glucose or $\mathrm{P}_{\mathrm{CO} 2}$, were imputed using the median value, as most of these features did not follow a normal distribution. Median and mode approaches were used to build imputation models and fill the missing values in the training set, and these imputation models were applied to the test set separately to avoid a data leakage problem.

\section{Generation of New Variables Through Data Transformation}

We performed minimum-maximum normalization to normalize numerical data for the ANN and logistic regression models. The minimum and maximum values of each feature from the training set were used to normalize the data in the training and test sets to avoid data leakage. Dummy encoding was performed on categorical features that contained more than 2 distinct values, such as pupillary reaction, but all categorical features with only 2 distinct values were dichotomized to accommodate them in the machine learning models. We used thresholds defined by PRISM-III to define normal and abnormal values. PIM-2 does not have defined thresholds; however, it penalizes any diversion of a vital sign from its normal value continuously.

\section{Model Training}

We built an ANN model using the Keras framework on top of TensorFlow (Google Brain Team) in Python (Python Software Foundation), with training conducted in Jupyter notebook (IPython). The Python code files that were used to build the models and generate results are available in Multimedia Appendix 1. We used a grid approach to determine the optimum configuration while designing the neural network. We tested various configurations between 1 and 3 hidden layers and 8 and 32 neurons per hidden layer, with a rule-of-thumb approach to limit the number of hidden layer neurons to the neurons in the input layer. Through experimentation, we identified that a 2-layer ANN, with 32 nodes in the first hidden layer and 16 nodes in the second hidden layer, performed better than the other configurations we tested. Our final model consisted of 32 input features (consisting of the variables used in the PRISM-III [1] and PIM-2 [2] models; see the Study Data Set section), and the 2 hidden layers, with each node using rectifier linear unit activation functions; finally, a sigmoid activated dense layer was used to predict the mortality for each instance (Figure 1). The model was compiled using an adam optimizer with a binary cross-entropy loss function. While keeping the main network the same, we also evaluated the model with unnormalized data as well as a model with only a single hidden layer. We conducted training with a batch size of 32 and observed that the loss remained constant after 100 epochs. 
Figure 1. Artificial neural network architecture with two hidden layers: the node in input layer "iXy" processes data from pediatric intensive care unit admission "X" with feature " $y$ " (such as age, length of stay, pupillary reaction, etc). The total number of features in the data set is denoted by "n." The first and second hidden layers are represented by h1 and h2, respectively, with a subscript to denote the node number. The output layer has a single node (o), which shows probability of mortality for patient "X." ReLU: rectifier linear unit.

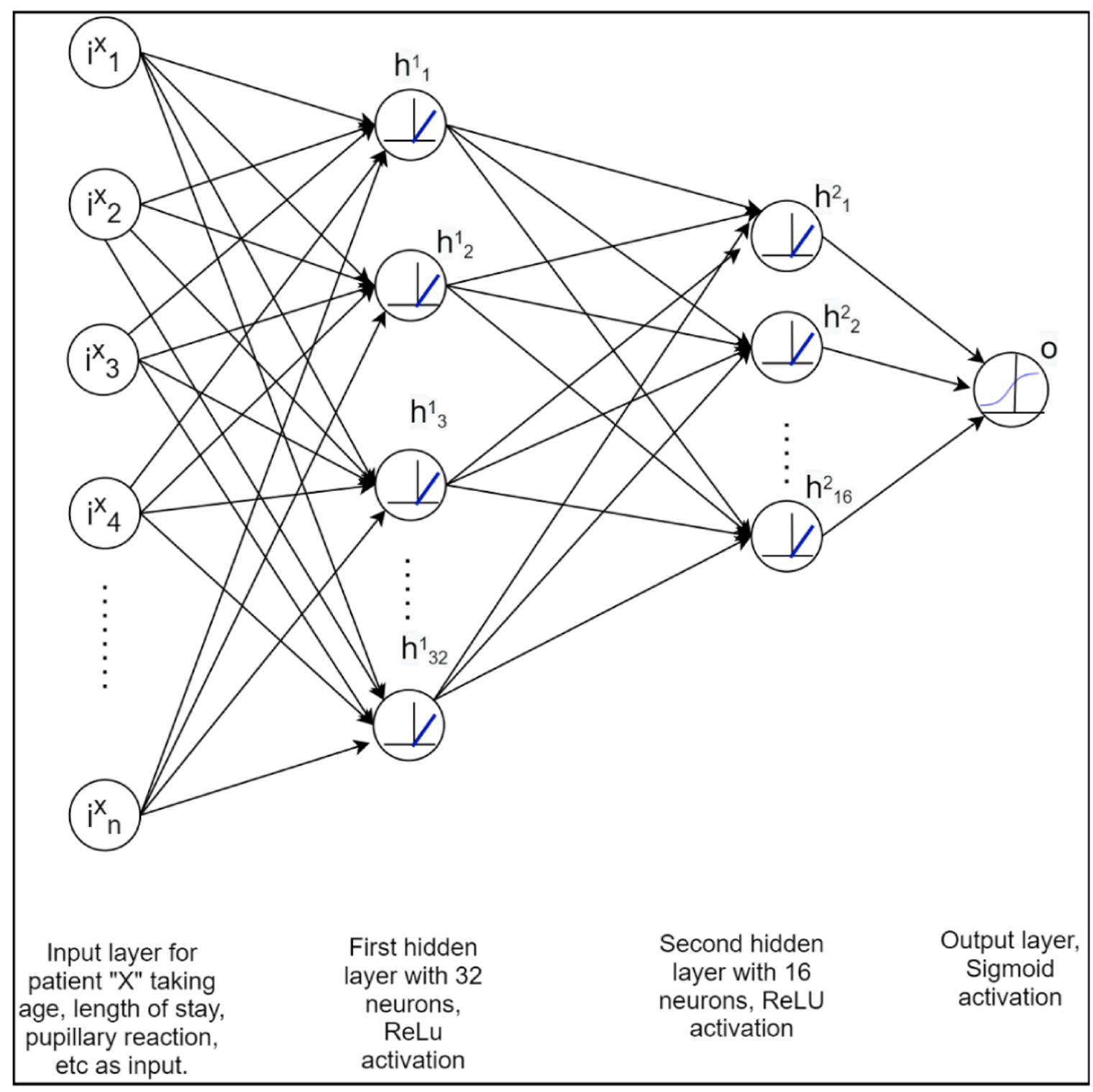

The ANN model was trained with features used in the PIM-2 and PRISM-III models to predict the outcome (died or survived); AUROC was used as an evaluation metric while training the model. Finally, we developed a logistic regression model for comparison using the same features from PIM-2 and PRISM-III.

\section{Model Evaluation}

The empirical range of AUROC scores was computed for each test set (obtained from bootstrap) using the sklearn.metrics function in Python. The test set that resulted in the median AUROC value was used to determine the optimum Youden index value. This threshold was then used to calculate the false positive rate (FPR) and false negative rate (FNR) for each test set, and the $95 \%$ empirical CIs were reported by pooling the results from all the test sets [15-17]; median and ranges of pooled results were reported for all other indices. We also reported the area under the precision recall curve (AUPRC) and its empirical $95 \%$ CI for each model. A Welch 2-sided $t$ test was used to compare AUROC and AUPRC for model pairs.
To compare how the models performed at specific true positive rate (TPR) and FPR levels, we fixed the TPR values at 95\%, $90 \%$, and $85 \%$ and computed the corresponding median FPR values (from all the test sets) for ANN, logistic regression, PIM-2, and PRISM-III. Similarly, we also reported the median TPR results by fixing the FPR at $5 \%, 10 \%$, and $15 \%$.

\section{Results}

\section{Data Set Characteristics}

The data set included 102,945 children with infection admitted between 2009 and 2014, of whom 4068 died (3.95\% mortality rate). The training sets contained 72,061 children, of whom a median of 2852 (range 2790-2903) died, equivalent to a $3.96 \%$ mortality rate; the test sets contained 30,884 children, of whom a median of 1216 (range 1165-1278) died, equivalent to a $3.94 \%$ mortality rate (Table 1$)$. 
Table 1. Overview of study population with demographics and risk factors split by outcome $(\mathrm{N}=102,945)^{\mathrm{a}}$.

\begin{tabular}{|c|c|c|c|c|c|c|c|}
\hline \multirow[t]{2}{*}{ Characteristic } & \multirow[t]{2}{*}{$\operatorname{All}(\mathrm{n}=102,945)$} & \multirow{2}{*}{$\begin{array}{l}\text { Died } \\
(n=4068)\end{array}$} & \multirow{2}{*}{$\begin{array}{l}\text { Survived } \\
(\mathrm{n}=98,877)\end{array}$} & \multicolumn{2}{|l|}{ Training } & \multicolumn{2}{|l|}{ Testing } \\
\hline & & & & $\begin{array}{l}\text { Died } \\
(\mathrm{n}=2852)\end{array}$ & $\begin{array}{l}\text { Survived } \\
(\mathrm{n}=69,209)\end{array}$ & $\begin{array}{l}\text { Died } \\
(n=1216)\end{array}$ & $\begin{array}{l}\text { Survived } \\
(\mathrm{n}=29,668)\end{array}$ \\
\hline Males, n (\%) & $58,058(56.39)$ & $2186(53.73)$ & $55,872(56.5)$ & $1531(53.68)$ & $39,075(56.46)$ & $655(53.87)$ & $16,797(56.62)$ \\
\hline \multicolumn{8}{|l|}{ Age } \\
\hline $\begin{array}{l}\text { Age (months), median } \\
\text { (IQR) }\end{array}$ & $28.9(7.5-100.3)$ & $\begin{array}{l}39.3(7.0- \\
137.7)\end{array}$ & $\begin{array}{l}28.6(7.5- \\
98.7)\end{array}$ & $\begin{array}{l}37.8(6.9- \\
138.4)\end{array}$ & $28.4(7.3-98.4)$ & $\begin{array}{l}42.45(7.2- \\
135.25)\end{array}$ & $29.3(7.9-99.4)$ \\
\hline$<1$ month, $\mathrm{n}(\%)$ & $4733(4.6)$ & $342(8.41)$ & $4391(4.44)$ & $245(8.59)$ & 3108 (4.49) & $97(7.98)$ & $1283(4.32)$ \\
\hline $1-23$ months, $\mathrm{n}(\%)$ & $42,935(41.71)$ & $1400(34.41)$ & $41,535(42.01)$ & $980(34.36)$ & $29,195(42.18)$ & $420(34.54)$ & $12,340(41.59)$ \\
\hline $2-5$ years, $\mathrm{n}(\%)$ & $22,264(21.63)$ & 719 (17.67) & $21,545(21.79)$ & $510(17.88)$ & $14,967(21.63)$ & 209 (17.19) & $6578(22.17)$ \\
\hline $6-12$ years, $\mathrm{n}(\%)$ & $18,652(18.12)$ & $776(19.07)$ & $17,876(18.08)$ & $542(19)$ & $12,474(18.02)$ & $234(19.24)$ & $5402(18.21)$ \\
\hline $13-18$ years, n $(\%)$ & 14,353 (13.94) & 829 (20.39) & $13,524(13.68)$ & $574(20.13)$ & $9461(13.67)$ & $255(20.97)$ & $4063(13.69)$ \\
\hline$>18$ years, $\mathrm{n}(\%)$ & $8(0.01)$ & $2(0.05)$ & $6(0.01)$ & $1(0.04)$ & $4(0.01)$ & $1(0.08)$ & $2(0.01)$ \\
\hline \multicolumn{8}{|c|}{ Primary diagnosis category, n (\%) } \\
\hline Respiratory & $63,928(62.1)$ & $1404(34.51)$ & $62,524(63.23)$ & $968(33.94)$ & $43,696(63.14)$ & $436(35.86)$ & $18,828(63.46)$ \\
\hline Infectious & $12,288(11.94)$ & $1387(34.1)$ & $10,901(11.02)$ & $979(34.33)$ & $7588(10.96)$ & $408(33.56)$ & $3313(11.17)$ \\
\hline Neurological & $3589(3.49)$ & $162(3.98)$ & $3427(3.47)$ & $120(4.21)$ & $2447(3.54)$ & $42(3.45)$ & $980(3.3)$ \\
\hline Gastrointestinal & $2248(2.18)$ & $103(2.53)$ & $2145(2.17)$ & $79(2.77)$ & $1518(2.19)$ & $24(1.97)$ & $627(2.11)$ \\
\hline Dermatologic & $1769(1.72)$ & $30(0.74)$ & 1739 (1.76) & $18(0.63)$ & $1219(1.76)$ & $12(0.99)$ & $520(1.75)$ \\
\hline \multicolumn{8}{|c|}{ Location before PICU ${ }^{b}$ admission, $n(\%)$} \\
\hline Inpatient & $30,691(29.81)$ & $1752(43.07)$ & $28,939(29.27)$ & $1238(43.41)$ & $20,280(29.3)$ & $514(42.27)$ & $8659(29.19)$ \\
\hline Postoperative admission & $18,435(17.91)$ & $576(14.16)$ & $17,859(18.06)$ & $412(14.45)$ & $12,447(17.98)$ & $164(13.49)$ & $5412(18.24)$ \\
\hline \multicolumn{8}{|l|}{ Resuscitation procedures } \\
\hline $\begin{array}{l}\text { Cardiac massage before } \\
\text { PICU, } \mathrm{n}(\%)\end{array}$ & $1863(1.81)$ & $588(14.45)$ & $1275(1.29)$ & $419(14.69)$ & $864(1.25)$ & $169(13.9)$ & $411(1.39)$ \\
\hline $\begin{array}{l}\text { Mechanical ventilation } \\
\text { within } 24 \text { hours, } \mathrm{n}(\%)\end{array}$ & $53,903(52.36)$ & 3417 (84) & $50,486(51.06)$ & $2405(84.33)$ & $35,258(50.94)$ & $1012(83.22)$ & $15,228(51.33)$ \\
\hline $\begin{array}{l}\text { Mechanical ventilation } \\
\text { within } 1 \text { hour, } \mathrm{n}(\%)\end{array}$ & $42,940(41.71)$ & $2658(65.34)$ & $40,282(40.74)$ & $1863(65.32)$ & $28,161(40.69)$ & $795(65.38)$ & $12,121(40.86)$ \\
\hline $\begin{array}{l}\text { Length of stay (days), me- } \\
\text { dian (IQR) }\end{array}$ & $3.5(1.7-8.0)$ & $\begin{array}{l}7.2(2.2- \\
21.4)\end{array}$ & $3.4(1.7-7.8)$ & $\begin{array}{l}7.1(2.2- \\
21.0)\end{array}$ & $3.4(1.7-7.8)$ & $\begin{array}{l}7.5(2.4- \\
22.7)\end{array}$ & $3.4(1.6-7.8)$ \\
\hline $\begin{array}{l}\text { PRISM-III }{ }^{\mathrm{c}} \text { probability of } \\
\text { death }(\%) \text {, median (IQR) }\end{array}$ & $0.63(0.3-1.6)$ & $10(1.7-44.6)$ & $0.5(0.3-1.4)$ & $\begin{array}{l}10.2(1.7- \\
47.8)\end{array}$ & $0.5(0.3-1.4)$ & $\begin{array}{l}8.3(1.6- \\
39.2)\end{array}$ & $0.5(0.3-1.4)$ \\
\hline $\begin{array}{l}\text { PIM- } 2^{\mathrm{d}} \text { probability of } \\
\text { death }(\%), \text { median (IQR) }\end{array}$ & $1(0.4-3.5)$ & $5.2(2.8-18)$ & $1(0.3-3.3)$ & $\begin{array}{l}5.3(2.9- \\
18.2)\end{array}$ & $0.9(0.3-3.3)$ & $\begin{array}{l}4.8(2.1- \\
17.6)\end{array}$ & $0.9(0.3-3.3)$ \\
\hline Died, n (\%) & $4068(3.95)$ & $4068(100)$ & $0(0)$ & $2852(100)$ & $0(0)$ & $1216(100)$ & $0(0)$ \\
\hline
\end{tabular}

${ }^{\mathrm{a}}$ Data are reported separately for the complete population and the training and test cohorts. Note that only the top 5 primary diagnosis categories are reported. In addition, note that only the initial 3 columns are true results; the remaining 4 are median values over the 100 data sets created.

${ }^{b}$ PICU: pediatric intensive care unit.

cPRISM-III: pediatric risk of mortality III.

${ }^{\mathrm{d}}$ PIM-2: pediatric index of mortality 2.

As is commonly encountered in large clinical registries using clinical availability of routinely collected data, between $0.41 \%$ $(424 / 102,945)$ and $80.27 \%(82,636 / 102,945)$ of entries were missing per feature required for PRISM-III: more commonly measured vital signs, such as systolic blood pressure and heart rate, had fewer missing values (424/102,945, $0.41 \%-517 / 102,945,0.5 \%$ ), whereas others were missing many entries, such as $\mathrm{CO}_{2}(49,837 / 102,945,48.41 \%$ missing) and partial thromboplastin time $(82,636 / 102,945,80.27 \%$ missing). For PIM-2, only $2.05 \%(2115 / 102,945)$ of entries were missing 
the numerical feature systolic blood pressure, whereas base excess was missing in $84.73 \%(87,230 / 102,945)$ of entries, and both fraction of inspired oxygen and $\mathrm{Pa}_{\mathrm{O} 2}$ were missing from $94.18 \%(96,958 / 102,945)$ of the entries. On the other hand, there was no missing information in any of the binary features, such as high- or low-risk diagnosis and recovery from surgery, which are features required for the PIM-2 calculation.

\section{Model Performance: ANN Trained Using Imputed and Normalized Data}

With the ANN trained on normalized data, the median FPR was mostly close to $18.4 \%$ (range $12.5 \%-30.8 \%$ ) and the median FNR value was $24 \%$ (range $12.7 \%-33.2 \%$; Table 2), with a median accuracy of $81.3 \%$ (range $69.9 \%-86.7 \%$ ) on the test set.

Table 2. Performance characteristics of 4 different mortality prediction models ${ }^{\mathrm{a}}$.

\begin{tabular}{llll}
\hline Prediction model & Threshold trigger $(\%)$ & False positive detections $\left(\mathrm{FPR}^{\mathrm{b}}\right), \mathrm{n}(\%)$ & ${\text { Missed cases }\left(\mathrm{FNR}^{\mathrm{c}}\right), \mathrm{n}(\%)}^{377(31.5)}$ \\
\hline PIM-2 $^{\mathrm{d}}$ & 3.36 & $7278(24.5)$ & $651(54.3)$ \\
PRISM-III $^{\mathrm{e}}$ & 2.21 & $1052(3.5)^{\mathrm{f}}$ & $317(26.8)$ \\
Logistic regression & 0.50 & $5142(17.3)$ & $289(24.0)^{\mathrm{f}}$ \\
ANN $^{\mathrm{g}}$ & 0.04 & $5467(18.4)$ & \\
\hline
\end{tabular}

${ }^{\text {a }}$ Comparison of the pediatric index of mortality 2, pediatric risk of mortality III, a traditional logistic regression model, and artificial neural network-based approach. For each model, the threshold was selected by optimizing the Youden index.

${ }^{b}$ FPR: false positive rate.

${ }^{\mathrm{c}}$ FNR: false negative rate.

${ }^{\mathrm{d}}$ PIM-2: pediatric index of mortality 2.

ePRISM-III: pediatric risk of mortality III

${ }^{\mathrm{f}}$ The best value in this category.

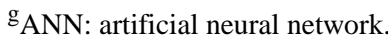

The AUROCs for PIM-2 and PRISM-III were 0.805 (95\% CI better than both PIM-2 $(P<.001)$ and PRISM-III $(P<.001$; Figure 0.801-0.816) and 0.844 (95\% CI 0.841-0.855), respectively. 2).

The ANN (AUROC 0.871, 95\% CI 0.862-0.880) performed

Figure 2. Receiver operating characteristic curves for four different mortality prediction models: pediatric index of mortality 2, pediatric risk of mortality III, logistic regression, and our best artificial neural network-based approach. The areas under the receiver operating characteristics curve and their 95\% CI are indicated in the bottom-right corner. ANN: artificial neural network; AUC: area under the receiver operating characteristic curve; LR: logistic regression; PIM-2: pediatric index of mortality 2; PRISM-III: pediatric risk of mortality III.

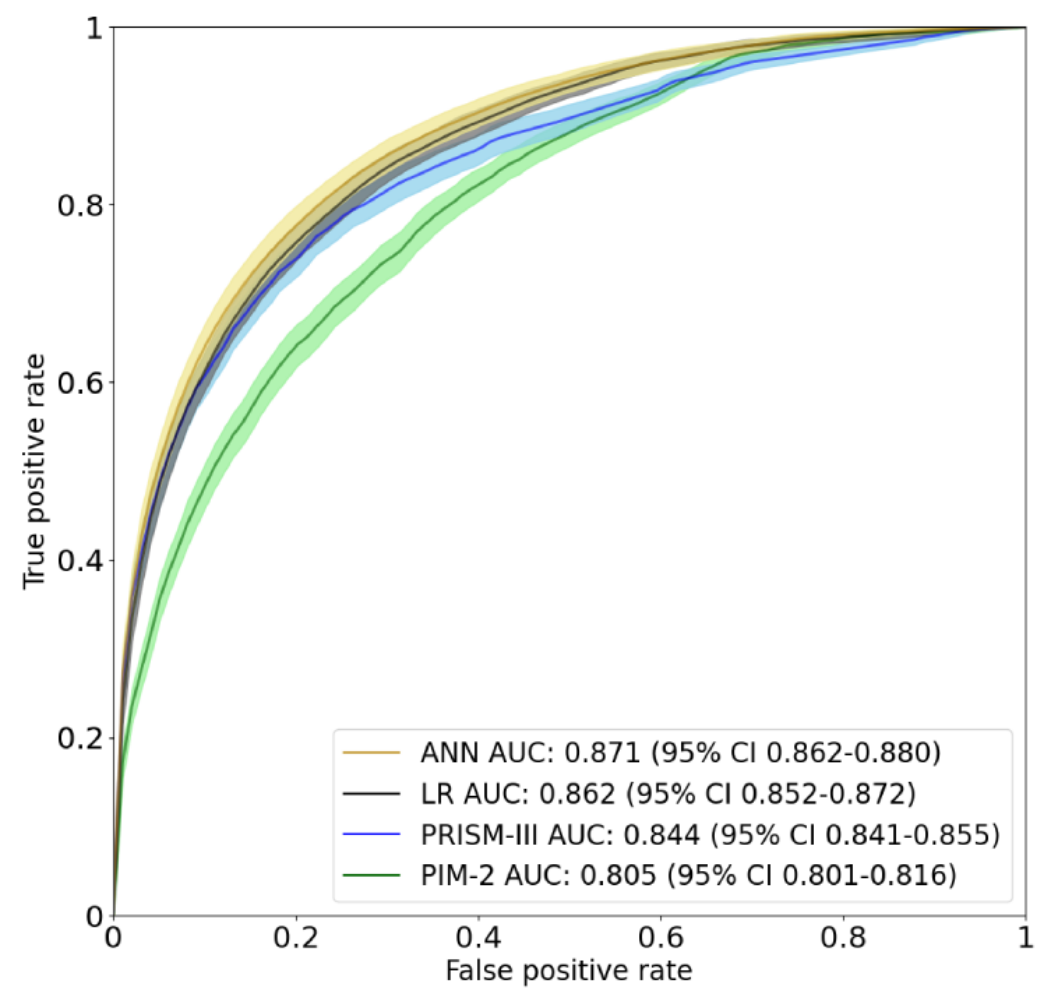


Similar results were observed using AUPRC, which indicated that the ANN (AUPRC 0.372, 95\% CI 0.345-0.396) performed better than PIM-2 (AUPRC 0.234, 95\% CI 0.213-0.255; $P<.001$ ) and PRISM-III (AUPRC 0.348, 95\% CI 0.322-0.367; $P<.001$; Figure 3). The ANN achieved the highest TPR compared with the logistic regression, PIM-2, and PRISM-III when FPR was fixed at $5 \%, 10 \%$, or $15 \%$. Similarly, FPR was lowest for the ANN when TPR was fixed at $85 \%$ or $90 \%$ (Table 3). However, the logistic regression model showed the smallest FPR when TPR was fixed at $95 \%$.

Figure 3. Precision recall curves for four different mortality prediction models: pediatric index of mortality 2, pediatric risk of mortality III, logistic regression, and our best artificial neural network-based approach. The areas under the precision recall curves and their 95\% CI, are indicated in the top right corner. ANN: artificial neural network; AUPRC: area under the precision recall curve; LR: logistic regression; PIM-2: pediatric index of mortality 2; PRISM-III: pediatric risk of mortality III.

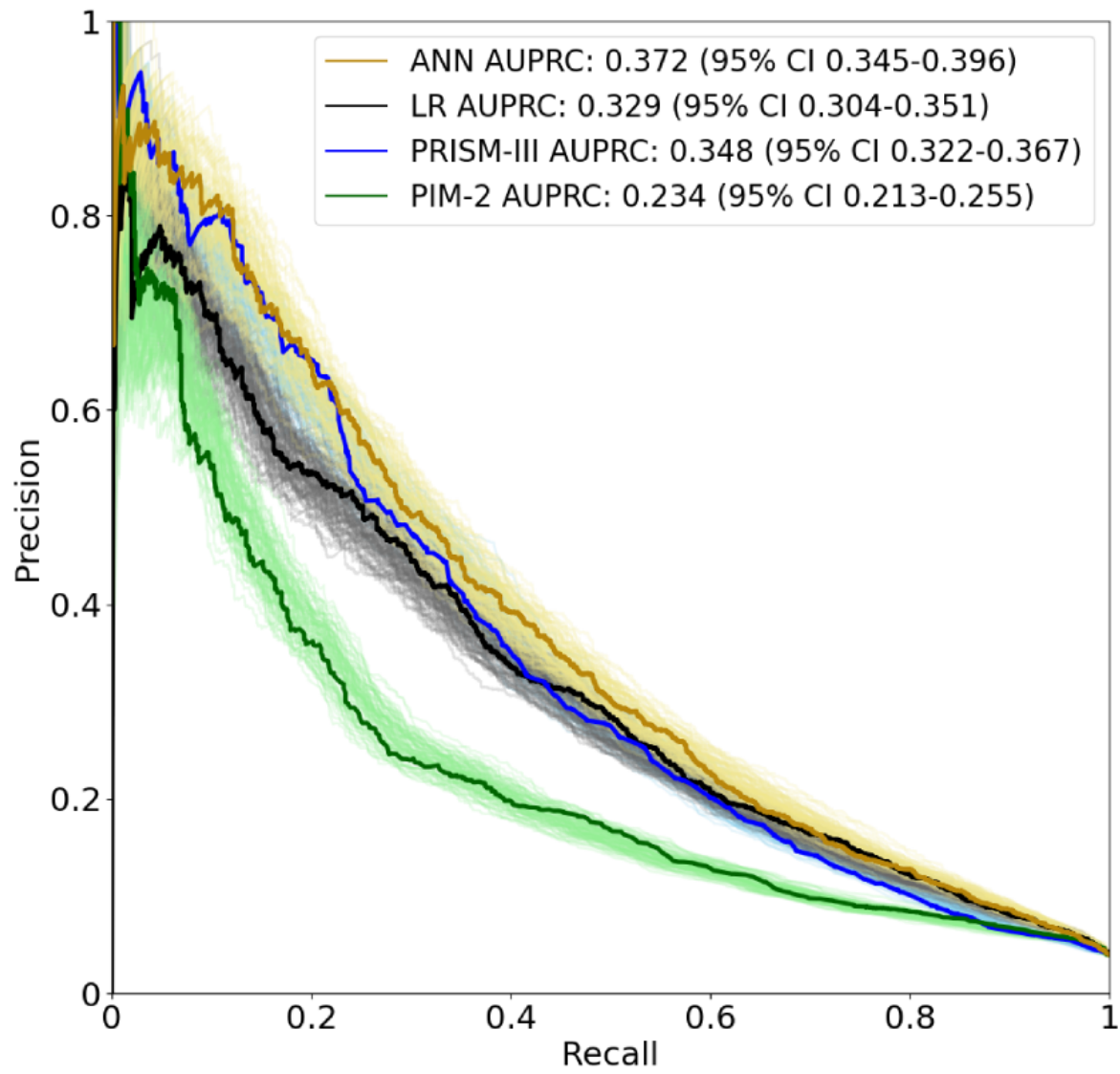

Table 3. Median true positive rate and median false positive rate of 4 different mortality prediction models ${ }^{\mathrm{a}}$.

\begin{tabular}{|c|c|c|c|c|c|c|}
\hline \multirow[t]{2}{*}{ Prediction model } & \multicolumn{3}{|l|}{$\mathrm{TPR}^{\mathrm{b}}(\%)$} & \multicolumn{3}{|l|}{$\operatorname{FPR}^{\mathrm{c}}(\%)$} \\
\hline & FPR fixed at $5 \%$ & FPR fixed at $10 \%$ & FPR fixed at $15 \%$ & TPR fixed at $95 \%$ & TPR fixed at $90 \%$ & TPR fixed at $85 \%$ \\
\hline PIM- $2^{\mathrm{d}}$ & 35.6 & 48.1 & 56.8 & 64.6 & 54 & 44.2 \\
\hline PRISM-III $^{\mathrm{e}}$ & 48.8 & 60.5 & 68.4 & 66.8 & 49.4 & 36.7 \\
\hline Logistic regression & 48.4 & 61.2 & 69.8 & $55.1^{\mathrm{f}}$ & 41.6 & 31.4 \\
\hline $\mathrm{ANN}^{\mathrm{g}}$ & $49.7^{\mathrm{f}}$ & $62.6^{\mathrm{f}}$ & $70.8^{\mathrm{f}}$ & 56 & $41.1^{\mathrm{f}}$ & $30.7^{\mathrm{f}}$ \\
\hline
\end{tabular}

${ }^{\mathrm{a}}$ Comparison of the pediatric index of mortality 2, pediatric risk of mortality III, a traditional logistic regression model, and artificial neural network-based approach.

$\mathrm{b}_{\text {TPR: true positive rate. }}$

${ }^{\mathrm{c}}$ FPR: false positive rate

${ }^{\mathrm{d}}$ PIM-2: pediatric index of mortality 2 .

${ }^{\mathrm{e}}$ PRISM-III: pediatric risk of mortality III.

${ }^{\mathrm{f}}$ The best value in this category.

${ }^{\mathrm{g}} \mathrm{ANN}$ : artificial neural network. 
The lowest FPR observed at the Youden-optimized threshold point for any of the models evaluated was $3.5 \%$ using PRISM-III, with a corresponding FNR of $54.3 \%$ (Table 2). If we target an FPR of $3.5 \%$, the corresponding FNRs for the other models were $68.7 \%$ for PIM-2, 55.8\% for the logistic regression, and $54 \%$ for the ANN.

\section{Model Performance: Logistic Regression Using Imputed and Nonnormalized Data}

The accuracy of the logistic regression model was $81.9 \%$ (range 81.4\%-82.5\%), with an FPR of $17.3 \%$ (range 17\%-18.3\%) and an FNR of $26.8 \%$ (range $23.7 \%-29.9 \%$; Table 2). The AUROC was 0.862 (95\% CI 0.852-0.872) and the AUPRC was 0.329 (95\% CI 0.304-0.351). The logistic regression model also showed better performance, as measured by AUROC, than both PIM-2 $(P<.001)$ and PRISM-III $(P<.001$; Figure 2$)$, but PRISM-III performed better than the logistic regression model when evaluated using AUPRC (Figure 3).

Although the AUROCs of the ANN and logistic regression overlap, it was found that ANN performed better than logistic regression $(P<.001)$.

\section{Model Performance: ANN Trained Using Imputed and Nonnormalized Data}

The accuracy of the ANN model trained using the nonnormalized data set with imputed data was $82.5 \%$ (range 69.6\%-89.2\%). The FPR value was $17.9 \%$ (range 9.7\%-31.1\%), and the FNR value was $26.7 \%$ (range $13.9 \%-39.3 \%$; Table 2). The AUROC was 0.865 (95\% CI 0.856-0.874), which was lower than that of the model with normalized data $(P<.001)$. The AUPRC value was 0.355 (95\% CI 0.328-0.376).

Using nonnormalized data, the ANN model had an FPR of $16.8 \%(95 \%$ CI $14.8 \%-18.2 \%)$ at a TPR of $73.3 \%$ and achieved its highest TPR of $73.4 \%$ (95\% CI 71.4\%-75.6\%) for an FPR of $17.3 \%$.

\section{Discussion}

\section{Principal Findings}

\section{Summary of Results}

We created an ANN-based pediatric risk prediction score using the features included in PIM-2 and PRISM-III scores, which we trained on patients from a large North American multicenter pediatric cohort with presumed sepsis as identified by a discharge diagnosis of infection. The overall performance of the ANN model with binary cross-entropy loss was better than the PIM-2 and PRISM-III scores, with median AUROCs of 0.871 (ANN) versus 0.805 (PIM-2; $P<.001)$ and 0.844 (PRISM-III; $P<.001)$. It also performed better than a traditional logistic regression model that used the same features required by PIM-2 and PRISM-III. However, these performance gains may not represent a clinically significant improvement. Our evaluation of the ANN approach with a single hidden layer and nonnormalized data returned poorer results than the other models evaluated.

\section{Improved Performance, but Is It Relevant?}

Our highest performing ANN was significantly better, statistically, than PIM-2 and PRISM-III using the AUROC and AUPRC measures of performance. The ANN missed fewer cases than PIM-2, PRISM-III, and the logistic regression model (ie, the ANN had a lower FNR; Table 2) at their respective ideal thresholds, as determined by optimizing their respective Youden indices; however, its rate of false positive detections was higher than that of PRISM-III and marginally higher than that of the logistic regression model (ie, the ANN had a higher FPR) at these Youden-optimized thresholds. This may suggest an opportunity for further optimization and evaluation, but it should be noted that the ANN did not miss more cases than PRISM-III (ie, the ANN had an equivalent FNR) when the FPR was fixed at the value of $3.5 \%$ (PRISM-III's Youden-optimized threshold). A direct comparison between models is challenging given that model selection will depend to a large extent on the clinical context; in some settings, a single objective (eg, to minimize FPR) may be the overriding concern, whereas in other cases, a balance of multiple objectives may be required (eg, to minimize both FPR and FNR).

Despite limited performance gains and increased robustness, the improvement may not be clinically relevant and is unlikely to overcome the initial concerns that physicians might have about the new model. The limited performance gains were not surprising. Although studies have proposed that ANNs outperform logistic regression models $[12,18]$ or offer at least partially better performance [19], a recent systematic review of 71 studies found no superior performance of ANN over logistic regression models [20]. However, ANN-based models allow for the tuning of performance characteristics, which offers a potential advantage.

\section{Trust Issues as a Barrier to ANN Use in Risk Modeling}

The successful acceptance of AI-based risk models requires physicians' willingness to accept AI models and the interpretability of those models. Although clinically improved performance might help this case, trust is a key element in acceptance, which is built (or lost) in a dynamic and evolving process [13,21]. Our failure to demonstrate a significant improvement in clinical performance will not help overcome the barriers to adoption.

Future AI-based risk models may need to become more interpretable to find acceptance [14], and the higher the risk, the more interpretability is needed to earn the trust. Including clinicians and patients in the development of AI models may be a step toward promoting acceptability and interpretability. Certification and licensure for AI models might also help build trust in model-based risk scores [22,23]. Finally, it may be useful to assure the user that the model is a tool and not a replacement for the clinician [13].

\section{Challenges With Skewed Data}

The working data set was skewed: only $3.95 \%(4068 / 102,945)$ of instances had the outcome as died. Local minima are a problem frequently associated with imbalanced data sets, and customized learning algorithms, cost functions, or external approaches (ie, resampling the data set) can be used to help 
overcome this problem [24]. Some ANNs tended to predict (mostly) everyone as a survivor; given the overall mortality rate of the population $(4068 / 102,945,3.95 \%)$, even assuming every patient will survive results in an accuracy of approximately 96\%, but with an FPR of zero and an FNR of one. A traditional experimental setup with accuracy as an evaluation metric fails when building models with skewed data, as the models tend to be biased toward the majority class (here survived) [25]. This challenge can be addressed by modifying the cost function to maximize the AUROC of the model [25].

\section{Limitations}

The main limitation of this work is the fact that out of several ANN-based models evaluated, only 1 type learned to discriminate between survival and death of patients effectively. Despite attempts to address the root cause (imbalance of outcomes in the data set), this suggests that the approaches selected may not have been optimal and that further network types and designs should be considered in future approaches. Following the initial positive outcomes with this model, secondary training on a data set can be used to fine-tune the ANN model.

The information included in the new models was limited to risk factors from PIM-2 and PRISM-III. By creating new features such as vital sign combinations or ratios [26], which in principle can be emulated by adding hidden layers, one might be able to provide another significant performance boost to the model. However, this did not seem to be the case in a recent sepsis prediction competition [27], where novel methods or applications seemed to be more promising than the creation of new features.

Another limitation was the relatively low number of complete patient entries in the VPS data set. Given that VPS is a curated data set, the potential reasons for this likely stem from local practices, such as tests not being required for clinical management in particular cases or it being generally decided that recording the results of these tests is optional. Although it makes the creation and use of some modeling techniques more difficult, this is an unavoidable feature of real-world clinical data. Characterizing the missingness to inform modeling might offer a valuable approach, but such features may not be generalizable because they represent local patterns of practice. To use models without the filtering layer, simple imputation approaches were used; however, data were likely not missing at random, which invalidates some of the (median or mode imputation) approaches used. More sophisticated approaches for handling data missingness, such as multivariate imputation by chained equations, may yield better performance [28,29], as the substituted values are likely closer to specific cases than the overall population. Importantly, physicians should inform the treatment of missing values, which might boost confidence in the methods used. It might be possible to use a complete time series in an ANN instead of extreme values observed in a certain window, which could improve performance.

This study explored only a limited range of ANN design techniques. For example, we used rectifier linear unit activation in the hidden layers but did not evaluate the effect of other activation functions on model performance; similarly, we used the adam optimizer to identify the optimal ANN architecture but did not evaluate alternative optimizers. Thus, more exhaustive experimentation may yield improved performance results. Similarly, Youden index was used as a pragmatic approach to identify the optimal cut off by maximizing the models' true positive and true negative rates. However, selecting the appropriate operating point for clinical implementation should consider alternative approaches to finding the optimal threshold and would also require a more nuanced evaluation of clinical priorities, which might, for example, penalize missed cases over false positives.

A major limitation to the development of a new risk score is the lack of recognized clinically acceptable performance criteria to assess the utility of integrating ANN-based risk scores into daily clinical routines. In their absence, it is difficult to make a clear statement on the clinical utility of models with slightly better performance compared with existing risk scores.

\section{Conclusions}

This study examined the performance of ANN models over logistic regression-based models to estimate the risk of mortality in the PICU. A simple 2-layer ANN demonstrated better performance than traditional logistic regression, PIM-2, and PRISM-III; the statistically significant improvement in performance may not be clinically significant. Further work, including involvement of physicians in defining performance thresholds, better handling of data missingness, and possibly the use of more sophisticated ANN-modeling methods, will be required to achieve meaningful advances to guide decision-making in the care of critically ill children.

\section{Acknowledgments}

This study formed part of author NGP's master's thesis, for which he would like to thank Professor Jens Ehlers (Department of Electrical Engineering and Computer Science, Technische Hochschule Lübeck, Lübeck, Germany) for his guidance and input in supervising the thesis work. Data were provided by VPS, LLC. No endorsement or editorial restriction of the interpretation of these data or the opinions of the authors has been implied or stated. The authors wish to thank the VPS team for their support in obtaining the data. MG holds a Michael Smith Foundation for Health Research Scholar salary award, and RSS holds a Mitacs Postdoctoral Fellowship. This work was supported by a 2020 BC Children's Hospital Research Institute External Salary Recognition Award (to MG). 


\section{Authors' Contributions}

SM and MG designed the study and obtained ethical approval to conduct the research. NGP initially analyzed the data with guidance from MG, and RSS revised the analysis. NGP, NCW, RSS, and MG interpreted the findings and drafted the manuscript. All authors critically reviewed the manuscript and read and approved the final version.

\section{Conflicts of Interest}

None declared.

\section{Multimedia Appendix 1}

Zipped Python code files were used to build the models and generate the results.

[ZIP File (Zip Archive), 29 KB-Multimedia Appendix 1]

\section{References}

1. Pollack MM, Patel KM, Ruttimann UE. PRISM III: an updated Pediatric Risk of Mortality score. Crit Care Med 1996 May;24(5):743-752. [doi: 10.1097/00003246-199605000-00004] [Medline: 8706448]

2. Slater A, Shann F, Pearson G, Paediatric Index of Mortality (PIM) Study Group. PIM2: a revised version of the Paediatric Index of Mortality. Intensive Care Med 2003 Mar 23;29(2):278-285. [doi: 10.1007/s00134-002-1601-2] [Medline: 12541154]

3. Ong ME, Lee Ng CH, Goh K, Liu N, Koh Z, Shahidah N, et al. Prediction of cardiac arrest in critically ill patients presenting to the emergency department using a machine learning score incorporating heart rate variability compared with the modified early warning score. Crit Care 2012 Jul 21;16(3):R108 [FREE Full text] [doi: 10.1186/cc11396] [Medline: 22715923]

4. Marcin JP, Pollack MM. Review of the acuity scoring systems for the pediatric intensive care unit and their use in quality improvement. J Intensive Care Med 2007 Jun 30;22(3):131-140. [doi: 10.1177/0885066607299492] [Medline: 17562737]

5. Johnson AE, Ghassemi MM, Nemati S, Niehaus KE, Clifton D, Clifford GD. Machine learning and decision support in critical care. Proc IEEE 2016 Feb;104(2):444-466. [doi: 10.1109/jproc.2015.2501978]

6. Peters C, Murthy S, Brant R, Kissoon N, Görges M. Mortality risk using a pediatric quick sequential (Sepsis-related) organ failure assessment varies with vital sign thresholds. Pediat Crit Care Med 2018;19(8):394-402. [doi:

$10.1097 /$ pcc.0000000000001598]

7. Shann F, Pearson G, Slater A, Wilkinson K. Paediatric index of mortality (PIM): a mortality prediction model for children in intensive care. Intensive Care Med 1997 Mar;23(2):201-207. [doi: 10.1007/s001340050317] [Medline: 9069007]

8. Topol EJ. High-performance medicine: the convergence of human and artificial intelligence. Nat Med 2019 Jan 7;25(1):44-56. [doi: 10.1038/s41591-018-0300-7] [Medline: 30617339]

9. Jiang F, Jiang Y, Zhi H, Dong Y, Li H, Ma S, et al. Artificial intelligence in healthcare: past, present and future. Stroke Vasc Neurol 2017 Dec 21;2(4):230-243 [FREE Full text] [doi: 10.1136/svn-2017-000101] [Medline: 29507784]

10. Haenssle HA, Fink C, Schneiderbauer R, Toberer F, Buhl T, Blum A, Reader study level-Ilevel-II Groups, et al. Man against machine: diagnostic performance of a deep learning convolutional neural network for dermoscopic melanoma recognition in comparison to 58 dermatologists. Ann Oncol 2018 Aug 01;29(8):1836-1842 [FREE Full text] [doi:

10.1093/annonc/mdy166] [Medline: 29846502]

11. Myers PD, Scirica BM, Stultz CM. Machine learning improves risk stratification after acute coronary syndrome. Sci Rep 2017 Oct 04;7(1):12692 [FREE Full text] [doi: 10.1038/s41598-017-12951-x] [Medline: 28978948]

12. Aczon M, Ledbetter D, Ho L, Gunny A, Flynn A, Williams J. Dynamic mortality risk predictions in pediatric critical care using recurrent neural networks preprint. arXiv : Statistics - Machine Learning. 2017. URL: https://arxiv.org/abs/1701. $\underline{06675}$ [accessed 2021-07-31]

13. Siau K, Wang W. Building trust in artificial intelligence, machine learning, and robotics. Cut Bus Technol J 2018;31(2):53 [FREE Full text]

14. Ching T, Himmelstein DS, Beaulieu-Jones BK, Kalinin AA, Do BT, Way GP, et al. Opportunities and obstacles for deep learning in biology and medicine. J R Soc Interface 2018 Apr 04;15(141):20170387 [FREE Full text] [doi: 10.1098/rsif.2017.0387] [Medline: 29618526]

15. Bouckaert R, Frank E. Evaluating the replicability of significance tests for comparing learning algorithms. In: Dai H, Srikant R, Zhang C, editors. Advances in Knowledge Discovery and Data Mining. Berlin: Springer; 2004:3-12.

16. Kuhn M, Johnson K. Applied Predictive Modeling. New York, NY: Springer; 2013:1-600.

17. Cohen P. Empirical Methods for Artificial Intelligence. Cambridge, MA: MIT Press; 1995:1-422.

18. Shi H, Lee K, Lee H, Ho W, Sun D, Wang J, et al. Comparison of artificial neural network and logistic regression models for predicting in-hospital mortality after primary liver cancer surgery. PLoS One 2012 Apr 26;7(4):e35781 [FREE Full text] [doi: 10.1371/journal.pone.0035781] [Medline: 22563399]

19. Eftekhar B, Mohammad K, Ardebili HE, Ghodsi M, Ketabchi E. Comparison of artificial neural network and logistic regression models for prediction of mortality in head trauma based on initial clinical data. BMC Med Inform Decis Mak 2005 Mar 15;5(1):3 [ㅌREE Full text] [doi: 10.1186/1472-6947-5-3] [Medline: 15713231] 
20. Christodoulou E, Ma J, Collins GS, Steyerberg EW, Verbakel JY, Van Calster B. A systematic review shows no performance benefit of machine learning over logistic regression for clinical prediction models. J Clin Epidemiol 2019 Jun;110:12-22. [doi: 10.1016/j.jclinepi.2019.02.004] [Medline: 30763612]

21. Hengstler M, Enkel E, Duelli S. Applied artificial intelligence and trust-The case of autonomous vehicles and medical assistance devices. Technol Forecast Soc Change 2016 Apr;105:105-120. [doi: 10.1016/j.techfore.2015.12.014]

22. LaRosa E, Danks D. Impacts on trust of healthcare AI. In: Proceedings of the 2018 AAAI/ACM Conference on AI, Ethics, and Society. 2018 Presented at: AIES '18: AAAI/ACM Conference on AI, Ethics, and Society; February 2 - 3, 2018 ; New Orleans LA USA p. 210-215. [doi: 10.1145/3278721.3278771]

23. Proposed regulatory framework for modifications to Artificial Intelligence/Machine Learning (AI/ML)-based Software as a Medical Device (SaMD). US Food and Drug Administration. 2019. URL: https://www.fda.gov/media/122535/download [accessed 2021-07-31]

24. Estabrooks A, Jo T, Japkowicz N. A multiple resampling method for learning from imbalanced data sets. Computational Intell 2004 Feb;20(1):18-36. [doi: 10.1111/j.0824-7935.2004.t01-1-00228.x]

25. He H, Ma Y. Imbalanced Learning: Foundations, Algorithms, and Applications. Hoboken, NJ: Wiley; 2013:1-216.

26. Pawar R, Bone J, Ansermino M, Görges M. An algorithm for early detection of sepsis using traditional statistical regression modeling. Comput Cardiol 2019;46:1-4 [FREE Full text] [doi: 10.22489/cinc.2019.061]

27. Reyna MA, Josef CS, Jeter R, Shashikumar SP, Westover MB, Nemati S, et al. Early prediction of sepsis from clinical data. Crit Care Med 2020;48(2):210-217. [doi: 10.1097/ccm.0000000000004145]

28. Buuren SV, Groothuis-Oudshoorn K. mice: Multivariate Imputation by Chained Equations in R. J Stat Soft 2011;45(3):1-67. [doi: $10.18637 /$ jss.v045.i03]

29. Kenward MG, Carpenter J. Multiple imputation: current perspectives. Stat Methods Med Res 2007 Jul 02;16(3):199-218. [doi: 10.1177/0962280206075304] [Medline: 17621468]

\section{Abbreviations}

AI: artificial intelligence

ANN: artificial neural network

AUPRC: area under the precision recall curve

AUROC: area under the receiver operating characteristic curve

FNR: false negative rate

FPR: false positive rate

ICU: intensive care unit

PICU: pediatric intensive care unit

PIM-2: pediatric index of mortality 2

PRISM-III: pediatric risk of mortality III

TPR: true positive rate

TRIPOD: Transparent Reporting if a multivariable prediction risk model for Individual Prognosis or Diagnosis VPS: Virtual Pediatric Systems

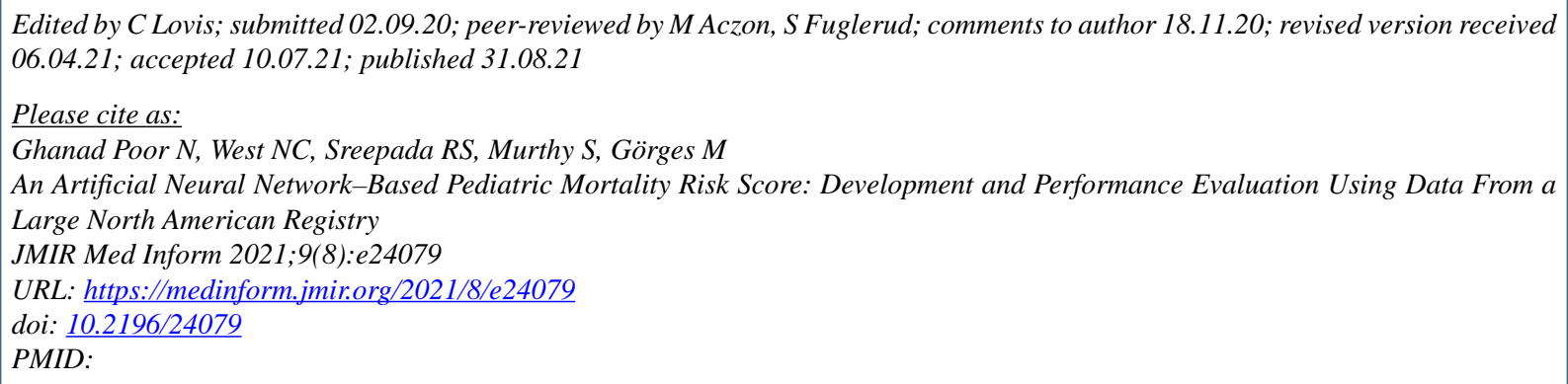

CNiema Ghanad Poor, Nicholas C West, Rama Syamala Sreepada, Srinivas Murthy, Matthias Görges. Originally published in JMIR Medical Informatics (https://medinform.jmir.org), 31.08.2021. This is an open-access article distributed under the terms of the Creative Commons Attribution License (https://creativecommons.org/licenses/by/4.0/), which permits unrestricted use, distribution, and reproduction in any medium, provided the original work, first published in JMIR Medical Informatics, is properly cited. The complete bibliographic information, a link to the original publication on https://medinform.jmir.org/, as well as this copyright and license information must be included. 